\title{
Resistencia de Mycobacterium tuberculosis a los fármacos antituberculosos en menores de 15 años en Colombia
}

\author{
Claudia Llerena1, Santiago Elías Fadul', María Consuelo Garzón¹, Graciela Mejía1, \\ Dora Leticia Orjuela1 ${ }^{1}$ Luz Mary García ${ }^{1}$, Hilda Beatriz Álvarez², Fernando Javier Ruiz \\ 1 Grupo de Micobacterias, Instituto Nacional de Salud, Bogotá, D.C., Colombia \\ 2 Laboratorio de Salud Pública de Antioquia, Secretaría de Salud y la Protección Social de Antioquia, Medellín, \\ Colombia \\ ${ }^{3}$ Laboratorio de Salud Pública de Atlántico, Secretaría de Salud de Atlántico, Barranquilla, Colombia
}

Introducción. La tuberculosis es una enfermedad infecciosa. Un tercio de la población mundial está infectada y cada año ocurren en el mundo nueve millones de casos, un millón en menores de 15 años y 719 en Colombia para el año 2008.

Objetivo. Describir el comportamiento de la resistencia de Mycobacterium tuberculosis en menores de 15 años en Colombia.

Materiales y métodos. Se analizó la información de las pruebas de sensibilidad de M. tuberculosis a los fármacos, realizadas desde 2001 hasta el 30 de junio de 2009 registradas en el Instituto Nacional de Salud. Se evaluó la distribución de casos por sexo, edad, localización de la enfermedad, tipo de muestra, infección concomitante tuberculosis/ $\mathrm{VIH}$, proporción de fármacorresistencia.

Resultados. Se incluyeron 128 pacientes, 66 (52\%) de sexo femenino y 62 (48\%) masculino. En el grupo de edad de 0 a 5 años, hubo 59 (46\%) casos, 21 (16,4\%) de 6 a 10 y 48 (37,5\%) de 11 a 14. Las formas de la enfermedad fueron: $89(69,6 \%)$ pulmonar, $34(26,4 \%)$ extrapulmonar y $5(3,94 \%)$ casos sin dato. Se encontraron $7(5,4 \%)$ casos con infección concomitante tuberculosis/VIH. Los casos no tratados fueron $123(96 \%)$ y $5(3,9 \%)$ fueron previamente tratados. La resistencia global en no tratados fue de $21,1 \%$ y la MDR-TB (multidrug-resistant tuberculosis) de 6,5\%.

Conclusiones. Este análisis demuestra que existen debilidades en el cumplimiento de las normas, lo que dificulta el diagnóstico y la identificación de los patrones de resistencia en menores de 15 años. Éste es un tema en el cual se debe explorar más.

Palabras clave: Mycobacterium tuberculosis, tuberculosis resistente a múltiples drogas, resistencia a medicamentos, agentes antituberculosos, tuberculosis pulmonar, tuberculosis latente, diagnóstico.

\section{Drug-resistant Mycobacterium tuberculosis in children under 15 years}

Introduction. Tuberculosis is a widespread and increasingly important infectious disease. A third of the world-wide population is infected. Every year nine million cases occur in the world, one million of them appearing in children under 15 years old. In Colombia alone, 719 cases were reported for the year 2008.

Objective. The incidence of Mycobacterium tuberculosis resistant forms was characterized in children under 15 years old in Colombia.

Materials and methods. Mycobacterium tuberculosis drug susceptibility test data from 2001 to mid year 2009 were analyzed at the Laboratorio Nacional de Referencia, Instituto Nacional de Salud, Bogotá, Colombia. Cases were evaluated by patient age, disease geographic location, and type of sample, as well as the proportions of TB/HIV coinfections and drug resistance.

Results. Of the 128 patients examined, 66 were female (52\%) and 62 were male (48\%). The age group 0 to 5 years old consisted of 59 cases (46\%), 21 cases were 6 to 10 years old (16\%) and 48 cases 11 to 14 years old $(37.5 \%)$. The types of disease were $89(69.6 \%)$ pulmonary, $34(26.4 \%)$ extrapulmonary, and $5(3.9 \%)$ cases without data. TB/VIH coinfection cases were $7(5.4 \%) .123(96 \%)$ untreated cases and $5(2.34 \%)$ cases previously treated. Global resistance among the untreated cases was $21.1 \%$ and MDR-TB was $6.5 \%$.

Conclusion. This analysis demonstrated weaknesses in following established procedures in the TB treatment process, thereby impeding the diagnosis and the identification of drug resistance $s$ in children under 15 years old. Approaches to improving treatment standards is a topic which requires further exploration.

Key words: Mycobacterium tuberculosis; tuberculosis, multidrug-resistant; drug resistance, antitubercular agents; tuberculosis, pulmonary; latent tuberculosis, diagnosis. 
La tuberculosis se incluye entre las enfermedades infecciosas más comunes y es una de las causas más frecuentes de muerte en todo el mundo. Aproximadamente, dos billones de personas, un tercio de la población mundial, está infectada por Mycobacterium tuberculosis (tuberculosis latente), cerca de nueve millones desarrollarán la enfermedad y cerca de un millón de los casos se presentan en menores de 15 años (1).

Según la Organización Mundial de la Salud (OMS), para el año 2006, la tasa de incidencia permaneció estable o disminuyó en todo el mundo; sin embargo, el número absoluto de casos nuevos se incrementó, en especial, en las regiones de África, Mediterráneo Oriental y Asia Suroriental (1).

En Colombia, la incidencia de tuberculosis ha presentando una tendencia sostenida a partir de 1997. Para 2008, la incidencia de todas las formas fue de 25,7 casos por 100.000 habitantes; el total de casos nuevos reportados fue 11.344, de los cuales, 7.196 presentaron baciloscopia positiva; los casos en menores de 15 años eran 719 , de los cuales, 269 (37,4\%) demostraron baciloscopia positiva $(2,3)$.

El diagnóstico de tuberculosis en menores de 15 años debe considerarse una actividad primordial. Un menor con enfermedad tuberculosa tiene mayor riesgo de progresión a formas graves, diseminación $y$, por consiguiente, mayor mortalidad, en especial, cuando por dificultades en el diagnóstico no hay un tratamiento efectivo (4).

En el año 2008, la OMS publicó el cuarto informe sobre el Proyecto Global de Vigilancia de Resistencia a los Fármacos Antituberculosos. Este documento contiene los datos de sensibilidad a los fármacos recopilados de 2002 a 2007 de 91.577 pacientes en 93 áreas geográficas de 81 países y 2 regiones administrativas de China. Este manuscrito no trata sobre la tendencia de la enfermedad en menores de 15 años (5).

La aparición de resistencia a los fármacos antituberculosos $\mathrm{y}$, en particular, la tuberculosis resistente a los medicamentos de primera línea (multidrug-resistant tuberculosis, MDR$T B$ ), definida como resistencia, por lo menos, a

Correspondencia:

Claudia Llerena Polo, Grupo de Micobacterias, Instituto Nacional de Salud, Avenida Calle 26 № 51-20, Bogotá, D.C., Colombia Teléfono: (571) 2207700 , extensión 1260

cllerena@ins.gov.co, claudiallerena@yahoo.com

Recibido: 17/12/09; aceptado:07/04/10 rifampicina e isoniacida, se ha convertido en un problema de salud pública y un obstáculo al control eficaz de la enfermedad. En muchos países se desconoce el grado de resistencia a los fármacos y es inadecuado el manejo de los enfermos de MDRTB. En quienes se haya demostrado resistencia, se deben tomar medidas específicas dentro de los programas de control para abordar el problema mediante el manejo apropiado de los pacientes y la adopción de estrategias para prevenir la aparición y la propagación de la enfermedad $(6,7)$.

La vigilancia de la resistencia a fármacos antituberculosos es esencial para disponer de información sobre la magnitud y la tendencia de la prevalencia de MDR-TB, lo que permite formular y evaluar las directrices del tratamiento y vigilar el efecto de las intervenciones. En el país, el Programa Nacional de Control de Tuberculosis tiene establecido que a toda muestra de un caso sospechoso de tuberculosis en un menor de 15 años se le debe hacer el diagnóstico por cultivo $y$, de ser positivo, pruebas de sensibilidad a los fármacos antituberculosos (8).

Además, el Laboratorio Nacional de Referencia del Instituto Nacional de Salud tiene establecidos los estándares bacteriológicos, según los cuales se deben cultivar las muestras pulmonares y extrapulmonares para diagnóstico de tuberculosis en menores de 15 años, casos de tuberculosis en población de alto riesgo $(\mathrm{VIH}$, diabetes, con trasplantes) y población vulnerable (indígenas, privados de la libertad, habitantes de la calle, escolares, fuerzas armadas y trabajadores de la salud), contacto sintomático respiratorio de un caso índice con resistencia a los fármacos antituberculosos, pacientes en control de tratamiento cuya baciloscopia sea positiva y aquellos casos que reingresan por abandono, recaída o fracaso del tratamiento $(8,9)$.

En el país se han realizado tres estudios de vigilancia de la resistencia de $M$. tuberculosis a los fármacos antituberculosos en pacientes no tratados. El primero, realizado en 1992, incluyó 829 pacientes, con una resistencia global de $14,1 \%$ y una MDR-TB de 1,8\% (10). El segundo estudio, realizado durante los años 1999 y 2000, incluyó 1.087 pacientes con una resistencia global de $15,6 \%$ y una MDR-TB de 1,5\% (11); en los años 2004 y 2005, en el tercero se evaluaron 925 casos no tratados con una resistencia global de $11,8 \%$ y una MDR-TB de $2,3 \%$; además, se evalúo la prevalencia de la resistencia en 264 casos 
previamente tratados; la resistencia global fue de $44,3 \%$ y la MDR-TB de $31,4 \%$ (12). Estos estudios no incluyeron casos de tuberculosis en menores de 15 años, debido a que las recomendaciones de la OMS y de la Organización Panamericana de la Salud (OPS) consideran como un criterio de exclusión el pertenecer a este grupo de edad (7,9-12).

El objetivo de este análisis es describir la resistencia de $M$. tuberculosis en menores de 15 años en Colombia, con base en la información del Laboratorio de Micobacterias del Instituto Nacional de Salud.

\section{Materiales y métodos}

Se hizo un estudio descriptivo retrospectivo de corte transversal.

Se consolidó, tabuló y analizó la información correspondiente a las pruebas de sensibilidad de M. tuberculosis a los fármacos antituberculosos estreptomicina, isoniacida, rifampicina y etambutol, realizadas desde el 2001 hasta 30 de junio de 2009 mediante la herramienta Microsoft Excel® 2007.

La metodología utilizada para determinar la sensibilidad de $M$. tuberculosis a los fármacos antituberculosos de primera línea fue la de proporciones de Canetti, Rist y Grosset, la cual está estandarizada por el Laboratorio Nacional de Referencia del Instituto Nacional de Salud y los Laboratorios de Salud Pública Departamentales de Atlántico y Antioquia $(13,14)$.

Se evaluó la distribución de casos por sexo, edad, localización de la enfermedad (pulmonar y extrapulmonar), tipo de muestra, infección concomitante tuberculosis/VIH, número de casos por entidad territorial de procedencia del cultivo, proporción de resistencia a cada uno de los fármacos de primera línea y la proporción de MDRTB. Además, se comparó el número de casos registrados al Programa Nacional de Control de Tuberculosis durante los años 2003 a 2008 con las pruebas de sensibilidad realizadas en este tiempo.

\section{Aspectos éticos}

Este documento es una revisión de la información que se recibe en el Laboratorio Nacional de Referencia del Instituto Nacional de Salud. Los aspectos éticos relacionados con los procesos de recolección de muestras en menores de edad siguieron las directrices del Comité de Ética de cada institución.

\section{Resultados}

En Colombia, los Laboratorios de Salud Pública Departamentales de Atlántico y Antioquia y el Instituto Nacional de Salud recibieron del 2001 al 30 de junio de 2009, 191 cultivos que correspondían a 128 casos en menores de 15 años. Se obtuvo el resultado de la prueba de sensibilidad en $133(70 \%)$ cultivos, $58(30 \%)$ no fueron aptos para realizar la prueba, debido a que algunos fueron negativos para bacilos ácido-alcohol resistentes (BAAR) o estaban contaminados, y otros presentaron bajo recuento o no fueron viables cuando se realizó la prueba de sensibilidad; no fue posible analizar la diferenciación de la causa de pérdida debido que no se contó con esta información.

Se encontró que 66 (52\%) de los casos analizados correspondían al sexo femenino y 62 (48\%) al masculino.

Al distribuirlos por edad, los casos se agruparon así: menores de un año, 14 (11\%); de 1 a menos de 2 años, 12 (9\%); de 2 a menos de 3 años, 9 (7\%); de 3 a menos de 4 años, 5 (4\%); de 4 a menos de 5 años, 9 (7\%); de 5 a menos de 6 años, 10 (8\%); de 6 a menos de 7 años, 7 (5\%); de 7 a menos de 8 años, 1 (1\%); de 8 a menos de 9 años, 5 (4\%), de 9 a menos de 10 años, 2 (2\%); de 10 a menos de 11 años, 6 (5\%); de 11 a menos de 12 años, 8 (6\%); de 12 a menos de 13 años, 9 (7\%); de 13 a menos de 14 años, 13 (10\%); y de 14 a menos de 15 años, 18 (14\%). Al agrupar los casos en rangos de edad, se encontró que de 0 a 5 años había 59 (46\%) pacientes, de 6 a 10 años, 21 (16,4\%), y de 11 a 14 años, 48 (37,5\%) casos (cuadro 1).

Del total de muestras analizadas, $89(69,6 \%)$ eran muestras pulmonares, 34 (26,4\%) eran extrapulmonares y en $5(3,9 \%)$ no fue posible establecer el tipo de tuberculosis (cuadro 2).

Las muestras de las cuales provenían los cultivos fueron: $43(33,6 \%)$ esputos, $40(31,3 \%)$ aspirados gástricos, 8 (6,3\%) líquidos cefalorraquídeos, $6(4,7 \%)$ lavados broncoalveolares, $6(4,7 \%)$ biopsias sin especificación del lugar de origen, 4 $(3,1 \%)$ biopsias de ganglio, $2(1,6 \%)$ biopsias de ganglio cervical, $2(1,6 \%)$ biopsias pleurales, 2 $(1,6 \%)$ secreciones sin especificación del lugar de recolección de la muestra, $1(0,8 \%)$ absceso de abdomen, 1 (0,8\%) biopsia ganglio axilar, 1 (0,8\%) biopsia hipocondrio, $1(0,8 \%)$ biopsia de piel, 1 $(0,8 \%)$ biopsia pulmonar, $1(0,8 \%)$ fistula de glúteo, $1(0,8 \%)$ líquido ascítico, $1(0,8 \%)$ líquido peritoneal, $1(0,8 \%)$ secreción mastoidea y $5(3,9 \%)$ cultivos sin información del tipo de muestra (cuadro 1). 
De los 59 casos que se encontraban en el grupo de 0 a 5 años, $38(6,4 \%)$ presentaban tuberculosis pulmonar. Las muestras obtenidas para su diagnóstico fueron de aspirado gástrico en 28 $(73,7 \%)$, de esputo en $7(18,4 \%)$ y de lavado broncoalveolar en 3 (7,9\%).

En el grupo de 6 a 10 años, hubo 21 casos, de los cuales, $13(61,9 \%)$ eran tuberculosis pulmonar. Las muestras obtenidas fueron: de aspirado gástrico en $3(23,1 \%)$, de esputo en $9(69,2 \%)$ y de lavado broncoalveolar en 1 (7,7\%).

En el grupo de 11 a 14 años se presentaron 48 casos, de los cuales 38 (79,2\%) eran formas pulmonares, que correspondieron a muestras de aspirado gástrico en 9 (18,8\%), de esputo en 27 $(56,3 \%)$ y de lavado broncoalveolar en $2(4,2 \%)$ (cuadro 2).
La infección concomitante tuberculosis/VIH se encontró registrada en $7(5,4 \%)$ pacientes, cuyas edades oscilaron entre 2 y 14 años, y las muestras se tomaron de diferentes sitios del cuerpo. Uno de estos casos presentaba MDR-TB (cuadro 3).

La procedencia de los cultivos según la entidad territorial fue: Valle, 33 (26\%); Antioquia, 26 (20,3\%); Bogotá, 19 (15\%); Amazonas, 10 (8\%); Santander, 10 (8\%); Quindío, 4 (3\%); Atlántico, 3 (2\%); Cauca, 3 (2\%); Cundinamarca, $3(2 \%)$; Vaupés, 3 (2\%); Guaviare 2, (1,6\%); La Guajira, 2 $(1,6 \%)$; Meta, 2 (1,6\%); Nariño, 2 (1,6\%); Caldas, $1(0,78 \%)$; Casanare, 1 (0,78\%); Huila, 1 (0,78\%); Norte de Santander, 1 (0,78\%); Putumayo, 1 $(0,78 \%)$, y Risaralda, 1 (0,78\%). Los departamentos no mencionados corresponden al $33 \%$, los cuales no hicieron envío de cultivos al Instituto Nacional de Salud.

Cuadro 1. Distribución de los casos por edades y tipos de muestra.

\begin{tabular}{|c|c|c|c|c|c|c|c|c|c|c|c|c|c|c|c|c|c|c|c|c|c|c|c|c|c|}
\hline \multirow[t]{2}{*}{ Tipos de muestra } & \multicolumn{9}{|c|}{ Edad (meses) } & \multicolumn{14}{|c|}{ Edad (años) } & \multirow[b]{2}{*}{ Total } & \multirow[b]{2}{*}{$\%$} \\
\hline & 0 & 2 & 3 & 4 & 5 & 6 & 7 & 8 & 9 & 1 & 2 & 3 & 4 & 5 & 6 & 7 & 8 & 9 & 10 & 11 & 12 & 13 & 14 & & \\
\hline Absceso de abdomen & & & & & & & & & & & & & & & 1 & & & & & & & & & 1 & 0,8 \\
\hline Aspirado gástrico & 2 & & & & & 1 & & 3 & 1 & 5 & 5 & 1 & 4 & 6 & 1 & & & 1 & 1 & 3 & 1 & 4 & 1 & 40 & 31,3 \\
\hline Biopsia & & & & & & & 1 & & & & & 1 & & & 1 & & 2 & & 1 & & & & & 6 & 4,7 \\
\hline Biopsia de ganglio axilar & & & & & & & & & & 1 & & & & & & & & & & & & & & 1 & 0,8 \\
\hline Biopsia de ganglio cervical & & & & & & & & & & 1 & & & & & & & & & & & 1 & & & 2 & 1,6 \\
\hline Biopsia de hipocondrio & & & & & & & & & & & & & & & & & & & & & & 1 & & 1 & 0,8 \\
\hline Biopsia de piel & & & & & & & & & & & & & & & & & & & & & & & 1 & 1 & 0,8 \\
\hline Biopsia pleural & & & 1 & & & & & & & & & & & & & & & & 1 & & & & & 2 & 1,6 \\
\hline Biopsia pulmonar & & & & & & & & & & & & 1 & & & & & & & & & & & & 1 & 0,8 \\
\hline Biopsia de ganglio & & & & & & & & & & & & & & 3 & & & & & & 1 & & & & 4 & 3,1 \\
\hline Esputo & & & & & & & & & & 2 & & 1 & 4 & & 3 & 1 & 2 & 1 & 2 & 3 & 4 & 6 & 14 & 43 & 33,6 \\
\hline Fístula en glúteo & & & & & & & & & & & 1 & & & & & & & & & & & & & 1 & 0,8 \\
\hline Lavado broncoalveolar & & & & 2 & 1 & & & & & & & & & & 1 & & & & & & 1 & 1 & & 6 & 4,7 \\
\hline Líquido cefalorraquídeo & & 1 & & & & & 1 & & & 3 & & & & 1 & & & 1 & & & 1 & & & & 8 & 6,3 \\
\hline Líquido ascítico & & & & & & & & & & & & & & & & & & & & & 1 & & & 1 & 0,8 \\
\hline Líquido peritoneal & & & & & & & & & & & & & & & & & & & & & 1 & & & 1 & 0,8 \\
\hline Líquido pleural & & & & & & & & & & & & 1 & & & & & & & & & & & & 1 & 0,8 \\
\hline Secreción & & & & & & & & & & & 1 & & & & & & & & & & & & 1 & 2 & 1,6 \\
\hline Secreción mastoidea & & & & & & & & & & & 1 & & & & & & & & & & & & & 1 & 0,8 \\
\hline Sin dato & & & & & & & & & & & 1 & & 1 & & & & & & 1 & & & 1 & 1 & 5 & 3,9 \\
\hline Total & 2 & 1 & 1 & 2 & 1 & 1 & 2 & 3 & 1 & 12 & 9 & 5 & 9 & 10 & 7 & 1 & 5 & 2 & 6 & 8 & 9 & 13 & 18 & 128 & 100 \\
\hline
\end{tabular}

Fuente: base de datos, Grupo de Micobacterias, Instituto Nacional de Salud

Cuadro 2. Distribución de los casos según la forma de la tuberculosis (pulmonar o extrapulmonar).

\begin{tabular}{|c|c|c|c|c|c|c|c|}
\hline \multirow[t]{2}{*}{ Localización } & \multirow[t]{2}{*}{ Muestras } & \multicolumn{6}{|c|}{ Grupos de edad } \\
\hline & & $0-5$ & $\%$ & $6-10$ & $\%$ & 11-14 & $\%$ \\
\hline \multirow[t]{3}{*}{ Pulmonar } & Aspirado gástrico & 28 & 47,4 & 3 & 14,3 & 9 & 18,7 \\
\hline & Esputo & 7 & 11,9 & 9 & 42,8 & 27 & 56,3 \\
\hline & Lavado broncoalveolar & 3 & 5,1 & 1 & 4,8 & 2 & 4,2 \\
\hline Subtotal & & 38 & 64,4 & 13 & 61,9 & 38 & 79,2 \\
\hline Extrapulmonares & & 19 & 32,2 & 7 & 33,3 & 8 & 16,6 \\
\hline Sin dato & & 2 & 3,4 & 1 & 4,8 & 2 & 4,2 \\
\hline Total & & 59 & 100 & 21 & 88 & 48 & 100 \\
\hline
\end{tabular}

Fuente: base de datos, Grupo de Micobacterias, Instituto Nacional de Salud 
Cuadro 3. Infección concomitante tuberculosis/VIH.

\begin{tabular}{ccclll}
\hline Edad (años) & N & Sexo & Tipo de muestra & MDR-TB & Departamento \\
\hline 2 & 2 & M & Sin dato & No & Cundinamarca \\
& & M & Fístula de glúteo & No & Antioquia \\
& 3 & M & Esputo & No & Valle \\
& & M & Lavado bronquial & No & Antioquia \\
12 & 1 & M & Absceso abdominal & No & Antioquia \\
& 1 & M & Biopsia de ganglio & No & Antioquia \\
\hline
\end{tabular}

Fuente: base de datos, Grupo de Micobacterias, Instituto Nacional de Salud

De los 128 casos evaluados, sólo 5 (3,9\%) habían sido previamente tratados: 1 (20\%) fracasó por resistencia a los cuatro fármacos antituberculosos evaluados y $2(40 \%)$ presentaron recaída y $2(40 \%)$ fueron abandonos, todos con sensibilidad a los fármacos. Los otros 123 casos se consideraron como no tratados, 97 (78,9\%) de los cuales fueron sensibles a los cuatro fármacos; en éstos, la resistencia global fue de 21,1\% y la MDR-TB de 6,5\%. Dos de las cepas $(1,6 \%)$ de MDR-TB presentaron resistencia combinada para estreptomicina y etambutol, es decir, a los cuatro fármacos.

La mayor resistencia se encontró para la estreptomicina en $5,7 \%$, seguida de la isoniacida con $3,3 \%$, rifampicina $1,6 \%$ y etambutol $0,8 \%$. No se encontró resistencia en las combinaciones de: rifampicina más etambutol, rifampicina más etambutol más estreptomicina, ni etambutol más estreptomicina (cuadro 4).

De los casos registrados en el Programa Nacional de Control de Tuberculosis durante los años 2003 a 2008 , se encontró que, en promedio, en $2,4 \%$ se obtuvo un cultivo al cual se le practicó prueba de sensibilidad; para el año 2007 este porcentaje fue de 3,4\% y para 2008 aumentó a 5,3\% (cuadro 5).

\section{Discusión}

Las acciones de los programas de control de tuberculosis como problema de salud pública se han dirigido generalmente a los adultos; el abordaje de la enfermedad en menores de 15 años se dificulta por la falta de un conocimiento claro del problema y los inconvenientes en el diagnóstico. Debido a la poca contagiosidad de estos casos, es normal que el Programa Nacional de Control de Tuberculosis se concentre en la identificación de las fuentes de contagio, es decir, los adultos bacilíferos que mantienen la cadena de transmisión.

Para el año 2007, en el país se notificaron 593 casos en menores de 15 años, correspondientes a $5,3 \%$ del total de casos nuevos, de los cuales,
Cuadro 4. Resistencia a los fármacos antituberculosos en casos no tratados de tuberculosis infantil, Colombia, 2001-2009*.

\begin{tabular}{|c|c|c|}
\hline & $\mathbf{n}$ & $\%$ \\
\hline & 123 & \\
\hline Sensibilidad total & 97 & 78,9 \\
\hline Resistencia total & 26 & 21,1 \\
\hline \multicolumn{3}{|l|}{ Resistencia a un fármaco } \\
\hline Isoniacida & 4 & 3,3 \\
\hline Rifampicina & 2 & 1,6 \\
\hline Estreptomicina & 7 & 5,7 \\
\hline Etambutol & 1 & 0,8 \\
\hline \multicolumn{3}{|l|}{$\begin{array}{l}\text { Resistencia a isoniacida más rifampicina y } \\
\text { a otro medicamento }\end{array}$} \\
\hline Isoniacida más rifampicina & 1 & 0,8 \\
\hline Isoniacida más rifampicina más etambutol & 1 & 0,8 \\
\hline Isoniacida más rifampicina más estreptomicina & 4 & 3,3 \\
\hline $\begin{array}{l}\text { Isoniacida más rifampicina más etambutol más } \\
\text { estreptomicina }\end{array}$ & 2 & 1,6 \\
\hline \multicolumn{3}{|l|}{ Resistencia a isoniacida y a otro medicamento } \\
\hline Isoniacida más etambutol & 1 & 0,8 \\
\hline Isoniacida más estreptomicina & 2 & 1,6 \\
\hline Isoniacida más etambutol más estreptomicina & 0 & 0,0 \\
\hline \multicolumn{3}{|l|}{ Resistencia a rifampicina y a otro medicamento } \\
\hline Rifampicina más etambutol & 0 & 0,0 \\
\hline Rifampicina más estreptomicina & 1 & 0,8 \\
\hline Rifampicina más etambutol más estreptomicina & 0 & 0,0 \\
\hline \multicolumn{3}{|l|}{ Otra resistencia } \\
\hline Etambutol más estreptomicina & 0 & 0,0 \\
\hline \multicolumn{3}{|l|}{ Total de resistencia por medicamento } \\
\hline Resistencia a isoniacida & 15 & 12,2 \\
\hline Resistencia a rifampcina & 11 & 8,9 \\
\hline Resistencia a estreptomicina & 16 & 13,0 \\
\hline Resistencia a etambutol & 5 & 4,1 \\
\hline \multicolumn{3}{|l|}{ Multirresistencia } \\
\hline MDR-TB & 8 & 6,5 \\
\hline
\end{tabular}

* Información al 30 de junio de 2009

Fuente: base de datos del Grupo de Micobacterias, Instituto Nacional de Salud

el $45 \%$ tenía baciloscopia positiva. Para el 2008, fueron 719 casos correspondientes a $6,1 \%$ del total de casos nuevos, de los cuales, $38 \%$ con baciloscopia positiva. Esto evidencia un incremento en el número de casos en esta población debido, posiblemente, a la falta de fortalecimiento de las actividades de vigilancia, detección, diagnóstico y 
Cuadro 5. Casos de tuberculosis en menores de 15 años registrados en el Programa Nacional de Control de Tuberculosis y pruebas de sensibilidad practicadas, años 2003 a 2008

\begin{tabular}{lccc}
\hline Año & $\begin{array}{c}\text { Casos } \\
\text { PNCT }\end{array}$ & $\begin{array}{c}\text { Prueba de } \\
\text { sensibilidad }\end{array}$ & $\begin{array}{c}\text { Porcentaje } \\
\text { de evaluados }\end{array}$ \\
\hline 2003 & 735 & 5 & 0,7 \\
2004 & 774 & 10 & 1,3 \\
2005 & 773 & 12 & 1,6 \\
2006 & 814 & 15 & 1,8 \\
2007 & 593 & 20 & 3,4 \\
2008 & 719 & 38 & 5,3 \\
Promedio & & & 2,4 \\
\hline
\end{tabular}

PNCT: Programa Nacional de Control de Tuberculosis

Fuente: Base de datos del Grupo de Micobacterias, Instituto Nacional de Salud

Archivos del Programa Nacional de Control de Tuberculosis, Ministerio de la Protección Social

tratamiento en adultos con formas infecciosas de la enfermedad, lo que favorece la transmisión a los menores de edad $(3,15)$.

La distribución de los casos por sexo no permite considerar que existan factores de riesgo asociados a la presencia de la enfermedad.

Al agrupar por edades los casos evaluados, el mayor aporte está en el grupo de 0 a 5 años (59 casos), lo cual ratifica la transmisión a partir de un adulto. El diagnóstico se hizo principalmente en muestras de aspirado gástrico, procedimiento invasivo que es, generalmente, el más utilizado; la siguiente muestra más frecuente fue de esputo $y$, finalmente, de lavado broncoalveolar. El grupo de 11 a 14 años presentó 48 casos. En ellos, la obtención de las muestras se facilita porque son enfermos bacilíferos, lo que aumenta la sensibilidad de los métodos diagnósticos de laboratorio. El grupo de 6 a 10 años presentó 21 casos. En estos dos grupos las muestras obtenidas fueron en su mayoría de esputo, seguido de aspirado gástrico y de lavado broncoalveolar.

El $69,5 \%$ de los casos evaluados eran pulmonares, la presentación más común de la enfermedad. El $26,6 \%$ correspondía a muestras extrapulmonares. Es importante resaltar que la sospecha de la enfermedad por un médico requiere un claro conocimiento de los signos y síntomas, aunque en muchas ocasiones son inespecíficos, así como de una buena correlación con los otros criterios diagnósticos, como el radiológico, la presencia de una reacción positiva de tuberculina y el epidemiológico, en el cual se valora el contacto cercano del menor con personas enfermas de tuberculosis $(15,16)$.
El diagnóstico microbiológico por cultivo y por baciloscopia se dificulta porque a menudo las muestras tienen poca carga bacilar, no es fácil su obtención en los menores de corta edad y falta calidad en el procesamiento de la muestra, lo que hace que disminuyan los resultados positivos. Sin embargo, siempre se debe intentar la comprobación bacteriológica, en especial, cuando hay formas graves de la enfermedad, pacientes infectados con VIH y casos con sospecha de MDR-TB (15).

A estas muestras se les debe realizar cultivo y, de ser positivo, prueba de sensibilidad a los fármacos como un aporte al diagnóstico, cumpliendo con las metodologías estandarizadas por el Laboratorio Nacional de Referencia del Instituto Nacional de Salud, así como los protocolos de los sistemas de gestión de la calidad que se llevan en los laboratorios de la Red Nacional de Laboratorios $(16,17)$.

Un estudio que se adelantó en hospitales de Bogotá y Medellín demostró que el proceso de recolección de las muestras permitía la confirmación bacteriológica en los niños cuando el procesamiento y la recolección era adecuados, lo que mejoraba la captación de casos por cultivo y disminuía los porcentajes de pérdida (Guerrero MI. Diagnóstico bacteriológico de la tuberculosis en menores de 15 años; Orozco LC. Viabilidad del M. tuberculosis expuesto al fosfato trisódico. Serie Memorias Eventos Científicos Colombianos. XII Congreso Colombiano de Laboratorio Clínico y II Congreso Nacional de Colbasan, 1985).

Se debe fortalecer el diagnóstico en toda la Red Nacional de Laboratorios, mediante la adquisición de equipos, como la centrífuga refrigerada que favorece la viabilidad del bacilo y mejora la confirmación bacteriológica, además de la posibilidad de practicar la prueba de sensibilidad y conocer la situación de resistencia a los fármacos antituberculosos en menores de 15 años $(17,18)$.

Los datos de infección concomitante demuestran que es un factor de riesgo poco investigado en menores de 15 años. El caso en un menor de dos años evidencia dificultades en la prevención del VIH en las madres y en la transmisión madre a hijo; sin embargo, el tema de la infección concomitante tuberculosis/VIH es algo que se debe mejorar en todo el país (19).

Al revisar el número de casos reportados al Programa Nacional de Control de Tuberculosis en menores de 15 años durante los años 2003 a 2008, se encuentra que el promedio de pacientes que 
obtuvieron un resultado de prueba de sensibilidad fue de $2,4 \%$. Esto indica que no se estaba cumpliendo con la norma (guía de atención integral). Durante los años 2007 y 2008 este porcentaje aumentó de $3,4 \%$ a $5,3 \%$, respectivamente, posiblemente debido al mayor número de casos con baciloscopia positiva y el aumento en el uso del cultivo para el diagnóstico $(8,9)$.

Con estos datos se evidencia que la resistencia sólo a la estreptomicina es de $5,7 \%$, la más alta, seguida de la isoniacida $(3,3 \%)$, la rifampicina $(1,6 \%)$ y el etambutol $(0,8 \%)$. Aunque la guía de atención integral de la tuberculosis indica que el manejo de casos en menores de 15 años debe hacerse sin la administración de estreptomicina, el hecho de que se presente mayor resistencia a este fármaco indica que se trata de una resistencia primaria trasmitida por un caso índice. Sin embargo, en estos casos es importante clarificar el antecedente de tratamiento, porque puede ser un caso que haya presentado una forma grave de la enfermedad, en el cual el uso de la estreptomicina es una opción en el manejo terapéutico $(20,21)$.

La vigilancia de la resistencia de $M$. tuberculosis en el país ha demostrado un aumento en el número de casos de MDR-TB en pacientes sin tratar. Algunos países pobres que presentan alta prevalencia de este tipo de la enfermedad registran una frecuente morbimortalidad en menores $(19,22)$.

Al comparar estos datos con los obtenidos en el tercer estudio nacional de vigilancia de la resistencia de $M$. tuberculosis a los fármacos antituberculosos (2004 a 2005), en pacientes no tratados se encontró que la proporción de resistencia a un solo fármaco es similar para la estreptomicina, la isoniacida y la rifampicina, aunque en este estudio no se reportó la resistencia sólo al etambutol. Sin embargo, este documento describe un pequeño número de casos, por lo cual sus datos no son significativos para evaluar la tendencia de la resistencia a los fármacos antituberculosos en este grupo de pacientes a nivel nacional $(7,12)$.

Se considera que en la información analizada existe sesgo, pues el criterio de inclusión para la prueba de sensibilidad en estos casos se orienta más hacia la presencia de la enfermedad, es decir, tuberculosis en menores de 15 años, y excluye otros criterios relevantes, como un tratamiento previo o infección con VIH.

El diagnóstico de MDR-TB se hace por laboratorio. Se debe sospechar cuando exista un caso contacto de un paciente con tuberculosis resistente a los medicamentos de primera línea; igualmente, cuando no se obtiene una respuesta adecuada al tratamiento antituberculoso o ante un antecedente previo de tratamiento. En estos casos se deben practicar cultivo y prueba de sensibilidad (15).

Cuando hay un paciente contacto con un caso documentado de tuberculosis farmacorresistente, se recomienda establecer el mismo esquema de tratamiento según la prueba de sensibilidad del contacto, estrategia que ha demostrado efectividad y oportunidad. Sin embargo, se debe hacer el cultivo para confirmar el patrón de resistencia (16). Estos casos se deben controlar mensualmente por clínica y por bacteriología; es primordial evaluar la ganancia de peso para ajustar las dosis del tratamiento $\mathrm{y}$, asimismo, detectar signos de toxicidad medicamentosa $(15,16,23-25)$.

Este análisis demuestra que existen debilidades en el cumplimiento de la normatividad vigente, que dificultan el diagnóstico y la identificación de los patrones de resistencia de las cepas de $M$. tuberculosis circulantes. Un consenso de la alianza "Alto a la TB" ha manifestado que el campo de la pediatría en MDR-TB es relativamente inexplorado y se deben conocer mejor las condiciones de seguridad y eficacia de los medicamentos, en especial, porque la literatura en este tema es limitada y existen pocas manifestaciones de reacciones adversas a los fármacos antituberculosos $(19,20)$.

Si se tiene en cuenta que el Programa Nacional de Control de Tuberculosis registró, para el año 2008, 269 casos de tuberculosis en menores de 15 años con baciloscopia positiva, se recomienda que el Ministerio de la Protección Social y el Instituto Nacional de Salud realicen un estudio de vigilancia de la resistencia a los fármacos antituberculosos, siguiendo las directrices de OMS/OPS, que permita conocer más de cerca la realidad de este problema (3).

Se requiere que el Programa Nacional de Control de Tuberculosis mejore la información disponible sobre tuberculosis en menores de 15 años y la notificación que se realiza a través del Sistema de Vigilancia en Salud Pública (Sivigila), para que se analicen los indicadores por grupos de edad y las cohortes de tratamiento $(9,26)$.

El país requiere una guía de manejo de los casos de tuberculosis en menores de 15 años enfocada en las actividades de detección, diagnóstico, tratamiento, seguimiento y prevención de farmaco- 
rresistencia, que pueda ser aplicada por los coordinadores del programa, así como por los especialistas en todo el territorio nacional.

Desde el Ministerio de la Protección Social se debe trabajar en vigilar el cumplimiento de las políticas públicas para la primera infancia, así como de los lineamientos contemplados en el Programa Ampliado de Inmunización y el de Atención Integral a Enfermedades Prevalentes de la Infancia, y maternidad segura; también, se debe trabajar en lograr la articulación con otras entidades, como el Instituto Colombiano de Bienestar Familiar, y el apoyo de la sociedad civil, que permitan el desarrollo de acciones conjuntas que fortalezcan el abordaje de la tuberculosis en menores de 15 años.

\section{Agradecimientos}

A Marcela Calle, representante de la Asociación Colombiana de Neumología Pediátrica, y a David Rodríguez, profesional del equipo nacional de tuberculosis, quienes conocen y están de acuerdo con los conceptos expresados en este documento; a ellos, mil gracias.

\section{Conflicto de intereses}

Los autores del artículo hacen constar que no existe, de manera directa o indirecta, ningún tipo de conflicto de intereses financieros, académicos o personales que puedan poner en peligro la validez de lo comunicado.

\section{Financiación}

Este trabajo fue financiado por el Instituto Nacional de Salud y la Secretaría de Salud de los departamentos de Antioquia y Atlántico.

\section{Referencias}

1. World Health Organization. Guidance for national tuberculosis programmes on the management of tuberculosis in children. Chapter 1: Introduction and diagnosis of tuberculosis in children. Int J Tuberc Lung Dis. 2006;10:109-17.

2. Castiblanco CA, Llerena C. Tuberculosis en Colombia: análisis de la situación epidemiológica, año 2006. Infectio. 2008;12:159-73.

3. Dirección General de Salud Pública, Ministerio de la Protección Social. Situación de la tuberculosis en Colombia durante el año 2008, documento en revisión para publicación. Bogotá: Ministerio de la Protección Social; 2009.

4. Martin J. Tuberculosis multirresistente en la infancia. Bol Pediatr. 2009;49:167-73.

5. World Health Organization. Global tuberculosis control -surveillance, planning, financing. Geneve: World Health Organization; 2009. p. 376.
6. Jaramillo E. La tuberculosis un reto que debemos enfrentar. Biomédica. 2004;24:5-8.

7. World Health Organization. Directrices para el tratamiento programático de la tuberculosis drogorresistente. Geneve: World Health Organization; 2006. p. 361.

8. Dirección General de Salud, Ministerio de Salud. Normas técnicas y guías de atención. Resolución 00412, febrero 25 de 2000. Guías de atención de la tuberculosis pulmonar y extrapulmonar. Bogotá: Ministerio de Salud; 2000. p. 1- 44.

9. Dirección General de Salud Pública, Ministerio de la Protección Social. Circular externa 058 de 2009. Lineamientos para el manejo programático de tuberculosis y lepra en Colombia. Bogotá, D.C.: Ministerio de la Protección Social; 2009.

10. Orozco LC, Aparicio G, Quintero O, Giraldo E, Ulloa I, León Cl. Resistencia de $M$. tuberculosis a los fármacos. Biomédica. 1981;1:130-4.

11. León $\mathrm{CI}$, Sierra C, Naranjo N, Garzón MC, Guerrero MI. Segundo estudio nacional de resistencia primaria de $M$. tuberculosis a las drogas antituberculosas en Colombia. Infectio. 2002;6:83.

12. Garzón MC, Angee DY, Llerena C, Orjuela D, Victoria J. Vigilancia de la resistencia de Mycobacterium tuberculosis a los fármacos antituberculosos, Colombia 2004-2005. Biomédica. 2008;28:319-26.

13. Canetti G, Rist N, Grosset J. Medida de la sensibilidad del bacilo tuberculoso a las drogas antituberculosas por el método de las proporciones. Buenos Aires: Dirección Lucha Antituberculosa; 1965.

14. Canetti G, Wallace F, Khomenko A, Mahler HT, Menon $\mathbf{N K}$, Rist $\mathbf{N}$, et al. Advances in techniques of testing mycobacterial drug sensitivity and the use of sensitivity tests in tuberculosis control programs. Bull World Health Organ. 1969;41:21-43.

15. Clemax C. The diagnosis of tuberculosis in childhood: update. Rev Peru Pediatr. 2007;60:105-10.

16. Starke JK. Tuberculosis in children. En: Reichman LB, Herschfield ES, editors. Tuberculosis. A comprehensive international approach. New York: Marcel Dekker Inc.; 1993. p. 329-67.

17. Abadco DL, Steiner P. Gastric lavage is better than bronchoalveolar lavage for isolation of Mycobacterium tuberculosis in childhood pulmonary tuberculosis. Pediatr Infect Dis J. 1992;11:735-8.

18. Garzón MC, Naranjo ON, Sierra CR, Llerena C, Orjuela DL. Bacteriología del Mycobacterium tuberculosis y de micobacterias no tuberculosas. Manual de procedimientos. Primera edición. Bogotá: Instituto Nacional de Salud; 2001. p. 20-1.

19. Espinal M, Reingold A, González G, Matos N, Romero R, Pérez G, et al. Infección por VIH y tuberculosis en niños. Arch Domin Pediatr. 1995;31:3-8.

20. García L, Vázquez F, Sanjurjo A, García JC. Transmisión horizontal de tuberculosis entre niños de una guardería. Rev Esp Salud Pública. 2000;74:573-5.

21. Bejarano N, Zarallo L, Cardesa J. Meningitis tuberculosa. Revisión epidemiológica y clínica de los pacientes 
ingresados en el hospital M-infantil de Badajoz durante los últimos 15 años. Vox Paediatrica. 2008;16:14-7.

22. Pérez $R$, Mellado $M$, Méndez $A$, Gillervelo $M$, García M, Villota $\mathrm{J}$, et al. Exposición a tuberculosis multidrogorresistente: estudio y seguimiento de nueve niños. An Pediatr. 2008; 68:490-5.

23. Schaaf S, Marais B, Whitelaw A, Hesseling A, Eley B, Hussey G, et al. Culture-confirmed childhood tuberculosis in Cape Town, South Africa; a review of 596 cases. BMC. 2007;7:140-8.
24. Drobac P, Mucherjee J, Joseph K, Mitnick C, Furn $\mathbf{J}$, Del Castillo $\mathbf{H}$, et al. Community-based therapy for children with multidrug-resistant tuberculosis. An Pediatr. 2006;117:2022-9.

25. Méndez A, Baquero F, García M, Rojo P, Ballesteros $Y$, Rubio B, et al. Tuberculosis multidrogorresistente en la edad pediátrica. An Pediatr. 2007;67:2006-211.

26. Dirección General de Salud Pública, Ministerio de la Protección Social. Decreto 3518 del 9 de octubre de 2006. Se crea y se reglamente el Sistema de Vigilancia en Salud Pública. Bogotá: Ministerio de la Protección Social; 2006. 